\title{
Resonance locking in giant planets indicated by the rapid orbital expansion of Titan
}

\author{
Valéry Lainey ${ }^{1,2 *}$, Luis Gomez Casajus ${ }^{3}$, Jim Fuller ${ }^{4}$, Marco Zannoni ${ }^{3}$, Paolo Tortora ${ }^{3}$, Nicholas \\ Cooper $^{5}$, Carl Murray ${ }^{5}$, Dario Modenini $^{3}$, Ryan Park $^{1}$, Vincent Robert ${ }^{2,6}$, Qingfeng Zhang ${ }^{7}$
}

\author{
${ }^{1}$ Jet Propulsion Laboratory, California Institute of Technology, \\ 4800 Oak Grove Drive, Pasadena, CA 91109-8099, United States \\ ${ }^{2}$ IMCCE, Observatoire de Paris, PSL Research University, CNRS, \\ Sorbonne Universits, UPMC Univ. Paris 06, Univ. Lille, 77 \\ ${ }^{3}$ Dipartimento di Ingegneria Industriale, Università di Bologna, 47121 Forlì, Italy \\ ${ }^{4}$ TAPIR, Mailcode 350-17, California Institute of Technology, Pasadena, CA 91125, USA \\ ${ }^{5}$ Queen Mary University of London, Mile End Rd, London E1 4NS, United Kingdom \\ ${ }^{6}$ IPSA, 63 bis boulevard de Brandebourg, 94200 Ivry-sur-Seine, France \\ ${ }^{7}$ Department of Computer Science, Jinan University, Guangzhou 510632, P. R. China
}

*To whom correspondence should be addressed; E-mail: lainey@imcce.fr.

Copyright 2019. All rights reserved. 
Saturn is orbited by dozens of moons, and the intricate dynamics of this complex system provide clues about its formation and evolution. Tidal friction within Saturn causes its moons to migrate outwards, driving them into orbital resonances that pump their eccentricities or inclinations, which in turn leads to tidal heating of the moons. However, in giant planets, the dissipative processes that determine the tidal migration timescale remain poorly understood. Standard theories suggest an orbital expansion rate inversely proportional to the power $11 / 2$ in distance ${ }^{1}$, implying negligible migration for outer moons such as Saturn's largest moon, Titan. Here, we use two independent measurements obtained with the Cassini spacecraft to measure Titan's orbital expansion rate. We find Titan rapidly migrates away from Saturn on a timescale of roughly $10 \mathrm{Gyr}$, corresponding to a tidal quality factor of Saturn of $Q \simeq 100$, which is more than a hundred times smaller than most expectations. Our results for Titan and five other moons agree with the predictions of a resonance locking tidal theor $y^{2}$, sustained by excitation of inertial waves inside the planet. The associated tidal expansion is only weakly sensitive to orbital distance, motivating a revision of the evolutionary history of Saturn's moon system. In particular, it suggests Titan formed significantly closer to Saturn and has migrated outward to its current position.

Prior monitoring of the mid-sized inner moons' orbital locations suggests that they are migrating outward faster than allowed if they formed at the same time as Saturn ${ }^{3,4}$, motivating new moon formation scenarios ${ }^{5,6}$. However, nearly all prior theoretical studies have assumed a constant tidal lag angle $\theta$ for the tidal bulge raised by each moon, parameterized by a tidal quality factor $Q \simeq 1 /(2 \theta)$. While the actual lag angle for tidally excited waves in the planet (dynamical tides) can vary, their effect can be described by an effective $Q$ value governing the tidal interaction with each moon, whose value is inversely proportional to the tidal energy 
dissipation rate within Saturn ${ }^{1}$. Denoting the semi-major axis $a$, the orbital expansion rate $t_{\text {tide }}^{-1}$ of each moon is

$$
t_{\text {tide }}^{-1}=\frac{\dot{a}}{a}=\frac{3 k_{2}}{Q} \frac{M_{\text {moon }}}{M}\left(\frac{R}{a}\right)^{5} n,
$$

where $M_{\text {moon }}$ is the mass of the moon, $R$ and $M$ are the radius and mass of Saturn, $k_{2}$ is the Love number of degree two that is determined by Saturn's density structure and is measured below, $n=2 \pi / P_{\text {orb }}=\sqrt{G M / a^{3}}$ is the moon's mean motion, and $P_{\text {orb }}$ is the moon's orbital period. Because of the strong dependence on $a$, most tidal theories predict slower migration for outer moons such as Titan.

To help explain the rapid migration of the mid-sized moons previously measured ${ }^{3,4}$, a new paradigm for the tidal evolution of moons, known as resonance locking ${ }^{7}$, was proposed ${ }^{2}$. Tidal dissipation due to inertial waves in Saturn's convective envelope ${ }^{8}$ or gravity modes in Saturn's deep interior ${ }^{9}$ is enhanced at discrete resonances with planetary oscillations. The resonant frequencies are determined by Saturn's internal structure, which is slowly evolving due to processes such as gravitational contraction, helium rain $^{10}$, and core erosion ${ }^{11}$. Moons can get caught in these resonances as Saturn's structure evolves, causing the moons to migrate outward on a timescale determined by Saturn's internal evolution. While explaining the fast orbital expansion of Rhea, the resonance locking theory predicted a similar expansion rate for Titan (and a smaller Saturnian tidal Q at Titan's frequency) ${ }^{2}$, making the monitoring of Titan's orbit a strong case for testing this model. Contrary to most tidal theories where the tidal $Q$ is constant for all moons, resonance locking predicts the tidal $Q$ for outer moons is much smaller.

To measure the migration rate of Titan, we use two independent methods. In the first approach, a coherent orbit of Titan was determined by reconstructing the trajectory of the Cassini spacecraft during 10 close encounters of the moon between February 2006 and August 2016. 
During each Titan encounter, we are sensitive to the relative position of the Cassini spacecraft with respect to both the moon and Saturn, providing indirect information on the orbit of Titan during the timespan of the Cassini mission. Our data sets encompass only radio tracking data acquired by the ground antennas of the Deep Space Network, namely Doppler observables at Xand Ka-band (8.4 GHz and $32.5 \mathrm{GHz}$, respectively), and range data at X-band. Due to limited temporal coverage of radiometric data in the vicinity of the other moons, it was not possible to obtain a reliable estimation of their orbits, which were instead retrieved from the latest satellite ephemerides released by JPL (see Methods).

The radiometric data analysis strategy was based on the classical approach used by the Cassini Radio Science Team in the past for gravity science experiments ${ }^{12-15}$. Our solution was obtained using JPL's orbit determination program MONTE $^{16}$, using a linearized weighted least squares filter that allowed us to determine corrections to an a-priori dynamical model taking into account all the relevant accelerations that affected the orbit of Titan and the trajectory of the Cassini spacecraft. The least squares information filter used a multi-arc approach, in which radiometric data obtained during non-contiguous orbital segments, called arcs, are jointly analyzed to produce a single solution of a set of global parameters, which affect all the arcs. Our global parameters include the initial state vector of Titan, its gravity field up to the 5th degree and order, Saturn's gravity field up to $J_{6}$, Saturn's tidal parameters $\operatorname{Re}\left(k_{2}\right)$ and $-\operatorname{Im}\left(k_{2}\right)=$ $k_{2} / Q \ll \operatorname{Re}\left(k_{2}\right)$ at Titan's frequency, and Cassini's thermal recoil acceleration.

Our second method is based solely on classical astrometry data. Similar to prior work ${ }^{4}$, we used more than a century of observations, starting in 1886 through the whole Cassini mission. New observations of the main moons ${ }^{17,18}$ were added to supplement previous data ${ }^{4}$. We also included for the first time extra observations of Titan from Imaging Science Subsystem 
(ISS) images, derived from Caviar software ${ }^{17}$, allowing for a tighter constraint on Titan's position during the Cassini mission. Our model solved the equations of motion of the eight main moons of Saturn, with the addition of the four Lagrangian moons of Dione and Tethys, as well as Pallene and Methone. Including Methone allows for a much better constraint on Mimas's orbital expansion, due to their proximity to mean-motion resonance. The Lagrangian moons are useful to obtain Saturn's Love number $k_{2}$, while Methone and Pallene are very sensitive to Mimas' mass and Saturn's gravity field. The perturbation of the four innermost moons of Saturn, Prometheus, Pandora, Janus and Epimetheus is introduced by ephemerides. We checked that the chaos affecting the orbits of these moons, as well as a possible secular variation of Saturn's $J_{2}$, did not affect our results (Methods).

In addition to the initial state vectors of the moons, we fitted the masses of the moons and their primary, the $J_{2}, J_{4}$ and $J_{6}$ of Saturn's gravity field, the orientation and precession of Saturn's pole, Saturn's $k_{2}$ (that assumes $k_{20}=k_{21}=k_{22}$ ), and Saturn's tidal ratio $k_{2} / Q$ at the tidal frequencies of Mimas, Enceladus, Tethys, Dione, Rhea and Titan. Due to the large uncertainty in Enceladus' current tidal dissipation rate ${ }^{19}$ estimated to be of order 15 GW [ref. $\left.{ }^{20}\right]$, we performed four independent fits, assuming a broad range of values of $3,10,33$ and 55 GW. Our results agree with prior measurements ${ }^{4}$, except for Tethys, as a consequence of the Mimas-Tethys mean-motion resonance and a different value for Mimas's migration rate.

Our findings are shown in Figure and Table 1. From the radio tracking data, we measure the tidal quality factor driving Titan's migration to be $Q=124_{-19}^{+26}$ ( $3 \sigma$ uncertainties), assuming a fixed $\operatorname{Re}\left(k_{2}\right)=0.382$ for Saturn. This corresponds to an outward migration rate of $11_{-2}^{+2} \mathrm{~cm} / \mathrm{yr}$. From astrometry, we find a slightly smaller value $Q=61_{-31}^{+240}$, but the two are consistent within $2 \sigma$ uncertainties. We tested the reliability of these results by performing 
many trials with different parameters (Methods), finding no substantial variation in our result. This unexpectedly small value of Saturn's $Q$ associated with Titan's migration is much smaller than our astrometric measurements of Saturn's $Q$ s associated with the other moons' migration, which range from $Q \sim 300$ for Rhea to $Q \gtrsim 3000$ for Tethys. The migration of each moon is clearly associated with a different value of $Q$ for Saturn, and all well-constrained values lie below the minimum value $Q=1.8 \times 10^{4}$ predicted if the moons formed at the same time as Saturn and $Q$ is constant ${ }^{21}$. Hence, our results show that most of Saturn's moons, including Titan, are migrating outward more rapidly than expected from classical tidal models.

The rapid migration of Titan is unexpected for all tidal dissipation mechanisms, except for resonance locking, which predicted the observed migration ${ }^{2}$. Figure shows the predicted tidal Qs (blue bars and points) for a resonance locking model with inertial waves with planetary spin evolution timescale $t_{p}=6 \mathrm{Gyr}$ (supplementary information), where the timescale $t_{p}$ is a parameter of the model that is expected to be comparable to the age of the solar system. The vertical extent of the blue bars of Mimas/Tethys and Enceladus/Dione is due to their mean-motion resonances, and accounts for the fact that the inner moon helps drive the outer moon's migration to an uncertain extent. ${ }^{2}$ In this model, the migration timescale of each moon is approximately

$$
t_{\text {tide }}=\frac{a}{\dot{a}} \approx \frac{3 t_{p}}{2}
$$

and is driven by the rate at which inertial wave "resonant" frequencies evolve along with Saturn's spin and structure. However, the predicted $Q$ is very different for each moon, with smaller values for outer moons, consistent with the trend in the data.

To quantify the net migration rate of each moon regardless of orbital resonances, we measure the difference in position between our best-fit solution, and one with no tidal dissipation in Saturn or Enceladus (Methods). Figure shows the corresponding migration time scale $t_{\text {tide }}$ 
Table 1: Estimated tidal parameters for Saturn. Top: Retrieved tidal parameters of the tidal bulge on Saturn raised by Titan, and their associated $3 \sigma$ uncertainties, using Cassini radio tracking data. Bottom: The same as the top table, including additional moons, based on astrometric data. The imaginary part of $k_{2}$ governs the migration of each moon. To account for tidal dissipation inside Enceladus, we performed four independent fits, assuming a heating rate of 3, 10, 33 and 55 GW. Error bars are $3 \sigma$ formal uncertainties. See also Supplementary Tables 1 and 2.

\begin{tabular}{c|c|c} 
Parameter & Value & Uncertainty \\
\hline $\operatorname{Re}\left(k_{2}\right)$ & 0.33 & 0.20 \\
$-\operatorname{Im}\left(k_{2}\right) \times 10^{4}$ & 30.8 & 5.5 \\
\hline
\end{tabular}

\begin{tabular}{c|c|c|c|c} 
Enceladus heating rate & $3 \mathrm{GW}$ & $10 \mathrm{GW}$ & $33 \mathrm{GW}$ & $55 \mathrm{GW}$ \\
\hline $\operatorname{Re}\left(k_{2}\right)$ & $0.382 \pm 0.017$ & $0.382 \pm 0.017$ & $0.382 \pm 0.017$ & $0.382 \pm 0.017$ \\
\hline$-I m\left(k_{2}\right) \times 10^{4}$ & & & & \\
Mimas & $0.54 \pm 0.99$ & $0.54 \pm 0.99$ & $0.54 \pm 0.99$ & $0.54 \pm 0.99$ \\
Enceladus & $1.19 \pm 0.46$ & $1.80 \pm 0.46$ & $3.32 \pm 0.50$ & $4.97 \pm 0.50$ \\
Tethys & $0.55 \pm 0.23$ & $0.55 \pm 0.23$ & $0.56 \pm 0.24$ & $0.55 \pm 0.23$ \\
Dione & $1.49 \pm 0.84$ & $1.38 \pm 0.84$ & $1.15 \pm 0.84$ & $0.88 \pm 0.84$ \\
Rhea & $14.0 \pm 3.4$ & $14.0 \pm 3.3$ & $14.1 \pm 3.4$ & $14.1 \pm 3.3$ \\
Titan & $69 \pm 57$ & $69 \pm 57$ & $69 \pm 57$ & $69 \pm 57$ \\
\hline
\end{tabular}


of each moon (Table 1 of supplementary material). Despite the different values of $Q$, the observed migration timescales for each of Saturn's moons tend to be similar, each consistent with $t_{\text {tide }} \approx 10 \mathrm{Gyr}$ within a factor of two, in line with the predictions of resonance locking.

By fitting the data with models of constant $Q$ or constant $t_{\text {tide, }}$, we show that a constant $Q$ model is ruled out with very high certainty, while a constant $t_{\text {tide }}$ model is more consistent with the data (Supplementary Table 1). Hence, we interpret our observations as strong evidence that a resonance locking process is driving the migration of many of Saturn's moons. The roughly constant migration time $t_{\text {tide }}$ for several of Saturn's moons suggests that resonance locking with inertial waves, rather than gravity modes (which predicts smaller $t_{\text {tide }}$ for outer moons), is the most probable explanation for the moons' migration (supplementary information). This may also help explain why mean-motion resonances between moons have survived, as resonance locking with gravity modes typically results in divergent migration that can disrupt mean-motion resonances between moons ${ }^{22}$, whereas resonance locking with inertial waves does not necessarily produce divergent migration and allows mean-motion resonances to survive.

While dissipation of waves within Saturn's convective envelope or stably stratified core ${ }^{23}$ seems to be the most significant mechanism of tidal friction, viscous dissipation of the quasistatic tidal bulge (i.e., equilibrium tidal dissipation) must also contribute. Our results in Figure indicate equilibrium tidal dissipation $Q_{e} \gtrsim 5000$ because this is the lowest allowable value for Tethys, but it remains possible that equilibrium tides govern the migration of Mimas, Enceladus, Tethys, and Dione.

Figure shows a possible orbital evolutionary history for Saturn's moons in the resonance locking framework, using a migration timescale for all moons of $t_{\text {tide }}=3 t_{\mathrm{Sa}}$, where $t_{\mathrm{Sa}}$ is the changing age of Saturn (supplementary information). This model is roughly consistent 
with our data, and it incorporates the fact that we expect shorter migration time scales in the past when Saturn was evolving more quickly. While mean-motion resonances and interaction with Saturn's rings (not accounted for here) can alter these histories, these models illustrate the qualitatively different behaviors of constant $Q$ and resonance locking models. This simple model predicts the semi-major axis evolves as $a \propto t^{1 / 3}$, though it necessarily breaks down as $t$ approaches zero. Our results indicate that Titan and Rhea have migrated much farther than previously expected, while the inner moons may have a markedly different history than they would in constant $Q$ models. The substantial migration of Titan may explain how it was able to capture Hyperion into mean-motion resonance ${ }^{24}$, and a previous resonance crossing with Iapetus may explain the latter's eccentricity and inclination ${ }^{25}$. Due to the changing values of $Q$ associated with resonance locking, Saturn likely had larger $Q$ values in the past, such that moons migrated more slowly than they would by assuming a constant $Q$. Hence, our results allow for low values of $Q$ for Saturn in the present day (and a correspondingly large tidal heating rate of Enceladus), even with an old age of the moons of at least a few Gyr, though it remains possible that the mid-sized moons formed after Saturn (supplementary information). Since our results favor resonance locking over constant $Q$ theories, orbital evolution calculations assuming that $Q$ is constant (either in space or in time ${ }^{6,26,27}$ ) should be revisited.

Resonance locking could operate in other moon systems, such as the Jovian system, where it might drive the outward migration of Io/Europa/Ganymede ${ }^{2}$ and predicts a much smaller effective $Q$ for Callisto if it is caught in a resonance lock. Resonance locking can also act in stellar binaries ${ }^{7,28}$ and exoplanetary systems, but it will not always dominate tidal dynamics, for instance, at very close separations when equilibrium tidal dissipation is more important, or when resonances are saturated by chaotic or non-linear effects ${ }^{29}$. But resonance locking could be especially important at wider separations where equilibrium tidal dissipation is negligible 
(as it is for Titan's migration), or in situations when a star or planet evolves on a relatively short time scale due to a rapid evolutionary phase, accretion, magnetic braking, or gravitational wave-driven inspiral ${ }^{30}$.

1. Goldreich, P. \& Soter, S. Q in the Solar System. Icarus 5, 375-389 (1966).

2. Fuller, J., Luan, J. \& Quataert, E. Resonance locking as the source of rapid tidal migration in the Jupiter and Saturn moon systems. Mon. Not. R. Astron. Soc. 458, 3867-3879 (2016).

3. Lainey, V. et al. Strong Tidal Dissipation in Saturn and Constraints on Enceladus' Thermal State from Astrometry. Astrophys. J. 752, 14 (2012).

4. Lainey, V. et al. New constraints on Saturn's interior from Cassini astrometric data. Icarus 281, 286-296 (2017).

5. Charnoz, S. et al. Accretion of Saturns mid-sized moons during the viscous spreading of young massive rings: Solving the paradox of silicate-poor rings versus silicate-rich moons. Icarus 216, 535-550 (2011).

6. Ćuk, M., Dones, L. \& Nesvorný, D. Dynamical Evidence for a Late Formation of Saturns Moons. Astrophys. J. 820, 97 (2016).

7. Witte, M. G. \& Savonije, G. J. Tidal evolution of eccentric orbits in massive binary systems. A study of resonance locking. Astron. Astrophys. 350, 129-147 (1999).

8. Ogilvie, G. I. \& Lin, D. N. C. Tidal Dissipation in Rotating Giant Planets. Astrophys. J. 610, 477-509 (2004).

9. Fuller, J. Saturn ring seismology: Evidence for stable stratification in the deep interior of Saturn. Icarus 242, 283-296 (2014). 
10. Salpeter, E. E. On Convection and Gravitational Layering in Jupiter and in Stars of Low Mass. Astrophys. J.l 181, L83 (1973).

11. Wilson, H. F. \& Militzer, B. Solubility of Water Ice in Metallic Hydrogen: Consequences for Core Erosion in Gas Giant Planets. Astrophys. J. 745, 54 (2012).

12. Tortora, P. et al. Rhea gravity field and interior modeling from Cassini data analysis. Icarus 264, 264-273 (2016).

13. Iess, L. et al. The Gravity Field and Interior Structure of Enceladus. Science 344, 78-80 (2014).

14. Iess, L. et al. Measurement and implications of saturn's gravity field and ring mass. Science 364, aat2965 (2019).

15. Durante, D., Hemingway, D. J., Racioppa, P., Iess, L. \& Stevenson, D. J. Titans gravity field and interior structure after Cassini. Icarus 326, 123-132 (2019).

16. Evans, S. et al. Monte: The next generation of mission design and navigation software. CEAS Space Journal 10, 79-86 (2018).

17. Cooper, N. J. et al. The Caviar software package for the astrometric reduction of Cassini ISS images: description and examples. Astron. Astrophys. 610, A2 (2018).

18. Zhang, Q. F. et al. First astrometric reduction of Cassini Imaging Science Subsystem images using an automatic procedure: application to Enceladus images 2013-2017. Mon. Not. R. Astron. Soc. 481, 98-104 (2018).

19. Choblet, G. et al. Powering prolonged hydrothermal activity inside Enceladus. Nature Astronomy 1, 841-847 (2017). 
20. Howett, C. J. A., Spencer, J. R., Pearl, J. \& Segura, M. High heat flow from Enceladus' south polar region measured using $10-600 \mathrm{~cm}^{-1}$ Cassini/CIRS data. Journal of Geophysical Research (Planets) 116, 3003 (2011).

21. Sinclair, A. T. A re-consideration of the evolution hypothesis of the origin of the resonances among Saturn's satellites. In Markellos, V. V. \& Kozai, Y. (eds.) IAU Colloq. 74: Dynamical Trapping and Evolution in the Solar System, vol. 106, 19-25 (1983).

22. Dermott, S. F., Malhotra, R. \& Murray, C. D. Dynamics of the Uranian and Saturnian satellite systems - A chaotic route to melting Miranda? Icarus 76, 295-334 (1988).

23. Fuller, J. \& Lai, D. Dynamical tides in compact white dwarf binaries: influence of rotation. Mon. Not. R. Astron. Soc. 444, 3488-3500 (2014).

24. Colombo, G., Franklin, F. A. \& Shapiro, I. I. On the formation of the orbit-orbit resonance of Titan and Hyperion. Astron. J. 79, 61 (1974).

25. Polycarpe, W. et al. Strong tidal energy dissipation in Saturn at Titan's frequency as an explanation for Iapetus orbit. Astron. Astrophys. 619, A133 (2018).

26. Crida, A. \& Charnoz, S. Formation of Regular Satellites from Ancient Massive Rings in the Solar System. Science 338, 1196- (2012).

27. Neveu, M. \& Rhoden, A. R. Evolution of Saturn's mid-sized moons. Nature Astronomy 3, $543-552$ (2019).

28. Fuller, J., Hambleton, K., Shporer, A., Isaacson, H. \& Thompson, S. Accelerated tidal circularization via resonance locking in KIC 8164262. Mon. Not. R. Astron. Soc. 472, L25-L29 (2017). 
29. Burkart, J., Quataert, E. \& Arras, P. Dynamical resonance locking in tidally interacting binary systems. Mon. Not. R. Astron. Soc. 443, 2957-2973 (2014).

30. Burkart, J., Quataert, E., Arras, P. \& Weinberg, N. N. Tidal resonance locks in inspiraling white dwarf binaries. Mon. Not. R. Astron. Soc. 433, 332-352 (2013).

Any correspondence should be addressed to lainey@imcce.fr

\section{Acknowledgments}

V.L.'s research was carried out at the Jet Propulsion Laboratory, California Institute of Technology, under a contract with the National Aeronautics and Space Administration. This work has been supported by the ENCELADE team of the International Space Science Institute (ISSI).

Support for this work was provided by the Italian Space Agency (L.G.C., M.Z., P.T., and D.M.) through the Agreement 2017-10-H.O in the context of the NASA/ESA/ASI Cassini/Huygens mission. J.F.'s research is funded in part by a Rose Hills Innovator Grant and the Sloan Foundation through grant FG-2018-10515. N.C. and C.M. were supported by the UK Science and Technology Facilities Council (Grant No. ST/M001202/1) and are grateful to them for financial assistance. N.C. thanks the Scientific Council of the Paris Observatory for funding. Q.Z.'s research was supported by the National Natural Science Foundation of China (Grant No. 11873026).

\section{Author Contributions}

All authors contributed to the writing of the manuscript. V.L. developed and fitted to the observations the full numerical model presented for the astrometric approach. P.T. led the radiometric 
data analysis approach. L.G.C. and M.Z. carried out the radiometric data analysis. J.F. provided theoretical interpretation, constructed figures, and performed supplementary calculations. D.M. contributed with software development. N.C., C.D., V.R. and Q.Z. provided extra astrometric data. R.P. provided extra expertise for astrometric analysis.

The authors declare no competing financial interests. 


\section{Methods}

Radiometric data selection and calibration To measure a precise orbit of Titan, we analyzed the Cassini radiometric data acquired during 10 close encounters with Titan (T11, T22, T33, T45, T68, T74, T89, T99, T110, and T122) throughout the Cassini mission. To increase the sensitivity we selected only the encounters with data coverage around the closest approach.

The main observable used in the reconstruction of Cassini's trajectory is the spacecraft range rate, obtained from the Doppler shift of a microwave carrier transmitted from ground at $\mathrm{X}$-band $(7.2 \mathrm{GHz})$ and sent back coherently at both $\mathrm{X}-(8.4 \mathrm{GHz})$ and $\mathrm{Ka}-\mathrm{band}(32.5 \mathrm{GHz})$. Doppler observables were integrated over a count time of $60 \mathrm{~s}$. In addition, in order to study the orbital evolution of Titan, we used range data at X-band.

We preferred two-way Doppler data over three-way, because of the intrinsic higher stability. When two-way observables were unavailable, we used three-way, adding in our filter the necessary bias to correct for possible DSN inter-station clock offset. When available, X/Ka measurements were preferred over $\mathrm{X} / \mathrm{X}$, as they are less sensitive to the dispersive effects, like Earth's ionosphere and solar and interplanetary plasma. We corrected the tracking data for the effects caused by the Earth's troposphere and ionosphere, using Global Positioning System data and microwave radiometer data, when available ${ }^{31}$. Tracking data acquired at ground station elevations lower than 15 degrees were discarded in order to avoid errors due to inaccurate calibration of the tropospheric and ionospheric induced delays. Furthermore, we generated corrections to take into account the additional Doppler shift induced by the spin of the Cassini spacecraft. 
Dynamical model The dynamical model included the relativistic point-mass gravitational acceleration from the Sun, the planets, the Moon, Pluto, and the main Saturn satellites. In addition, the setup included the gravity field of Saturn and its planetary rings resulting from the analysis of data from the Grand Finale orbits ${ }^{14}$. Saturn's response to the tides raised by Titan was modelled using a complex Love number $k_{2}$. Furthermore, the model included the following non-gravitational accelerations for Cassini: the solar radiation pressure, the drag induced by the upper-layer of Titan's atmosphere, and the acceleration due to the non-isotropic thermal emission, mainly generated by the three onboard Radioisotope Thermoelectric Generators.

No constraints were applied to the estimation of the global parameters, because the a priori uncertainties were chosen to be at least one order of magnitude larger than the obtained formal uncertainties or the formal uncertainty currently available from the literature ${ }^{4,14}$. Besides global parameters, we estimated also local parameters, which means that they affect only a single arc. For each encounter, they include the initial state of Cassini, the drag perturbation during the low-altitude flybys, the low gain antenna phase-centre position during T110, constant Doppler bias for the three-way passes, and constant range biases per station and pass. The a priori uncertainties for Cassini's position and velocity were $20 \mathrm{~km}$ and $0.2 \mathrm{~m} / \mathrm{s}$, respectively.

Measurement of Titan's orbit The inclusion of the Saturn's tidal dissipation at Titan's frequency to our dynamical model allowed for a fit to the noise level, as shown by the range-rate and range residuals (Supplementary Figures 1-4). The estimated gravity field of Titan and Saturn are both fully compatible with the latest measurements published by the Cassini RS team $^{14,15}$.

The radiometric data have proven to be very sensitive to the imaginary component of Saturn's Love number, $\operatorname{Im}\left(k_{2}\right)$, that drives tidal migration. During each encounter, we can 
accurately measure the relative position of the Cassini spacecraft with respect to Titan. In addition, outside the sphere of influence of Titan, we are sensitive to the relative position of the spacecraft with respect to the gas giant. As a result, the analysis of the data acquired during all close encounters provides information on the relative position of Titan with respect to Saturn. The formal uncertainty in the Titan's measured position is $3 \mathrm{~m}$ in the radial direction, almost constant during the timespan of the Cassini mission, while the positional uncertainty in the transverse direction has a minimum of $4 \mathrm{~m}$ in January 2010, and then grows almost linearly to a maximum of $40 \mathrm{~m}$.

The imaginary part of Saturn's Love number at Titan's frequency causes an orbital migration of the satellite in the radial and transverse position with respect to Saturn. Assuming $\mathrm{Q}=$ 100 , the total effect is about $1.1 \mathrm{~m}$ on the radial direction and $1.2 \mathrm{~km}$ on the transverse direction after 10 years. However, most of the longitudinal shifts will disappear when fitting for the satellites' orbits, so that the observable orbital signal associated with $\operatorname{Im}\left(k_{2}\right)$ corresponds to the part of the drift that cannot be absorbed. In order to compute the effect of Saturn's dissipation on Titan's orbit during the timespan of the Cassini mission we performed numerical simulations, following the same approach as prior work ${ }^{34}$. As a result of our simulations we found that the signal on Titan's longitude amounts to $150 \mathrm{~m}$. This distance is much larger than our formal uncertainty, making the effect of the dissipation clearly observable.

Robustness of the solution The stability of our solution has been assessed by carrying out several tests, including changing the values of the less observable $\operatorname{Re}\left(k_{2}\right)$, and the use of different ephemerides to take into account possible changes in the orbits of the other Saturn satellites. In all cases, the estimated values were compatible with the reference solution within $1 \sigma$, and they offered range-rate residuals of very similar quality. 
Another possible source of error may come from a time variation of $J_{2}$. In particular, a linear variation of $J_{2}$ produces a quadratic drift on the mean longitude, similar to the effects of Saturn's dissipation. Hence, in order to check the effect of this parameter on our results, we introduced a secular variation of Saturn's $J_{2}$ equal to $4.42 \times 10^{-13}$ day $^{-1}$, corresponding to the upper limit estimated by the astrometric fit (see next section). The estimated value of $\operatorname{Im}\left(k_{2}\right)$ remained compatible with our nominal solution within $1 \sigma$. In the analysis performed using radiometric observables, dissipation within Titan has been neglected. To check the robustness of the obtained solution to this effect, we introduced the $\operatorname{Im}\left(k_{2, T i}\right)$ of Titan in the list of parameters to solve for, retrieving a solution which is statistically equivalent. In this case, we found the value of $\operatorname{Im}\left(k_{2, T i}\right)$ of Titan (causing tidal dissipation within Titan) to be $\operatorname{Im}\left(k_{2, T i}\right)=-1.248 \times$ $10^{-2} \pm 4.479 \times 10^{-2}$, compatible with zero within 1-sigma.

Estimating the secular variation of Saturn's $J_{2}$ Since a secular variation of Saturn's $J_{2}$ would provide a secular effect on the moons' semi-major axis, we tried assessing its possible magnitude using astrometry data. For that matter, we considered Pan's orbit. Indeed, Pan orbits extremely close to Saturn and is not involved in any orbital resonance. Moreover, its small size allows for an almost perfect determination of its center of mass from the Imaging Science Subsystem (ISS)/Cassini images. Lastly, its small mass does not allow for a significant dynamical coupling with the Saturn's rings. Hence, Pan is a perfect candidate for probing a secular effect on $J_{2}$. The dynamical modeling we considered here is similar to the one presented in the main text, except that Daphnis was added as a perturber, and the orbits of the coorbitals as well as Methone and Pallene were discarded. All the moons (except Pan itself) were forced from ephemerides. We solved for the initial state vector of Pan, the polar orientation and precession of Saturn, and Saturn's gravity coefficients $J_{2}, J_{4}, J_{6}$, and $\dot{J}_{2}$. 
To check the potential influence of the choice of moons' ephemerides on the estimation of Saturn's $J_{2}$ from Pan's orbit, we considered three independent fits. The first one introduced sat382 and sat360xl for the ephemerides of the inner moons and the main moons respectively. The second one introduced sat382 for the inner moons and NOE-6-2018-MAIN ephemerides. These last ephemerides correspond to the solution in the present paper for assuming 33GW of tidal dissipation wihtin Enceladus. Our third test considered NOE-6-2018-inner-eph4 and NOE-6-2018-MAIN ephemerides for the inner and main moons, respectively. We obtained for these three independent fits the solutions $\dot{J}_{2}=(2.3 \pm 4.4) \times 10^{-13},(2.0 \pm 4.4) \times 10^{-13},(-1.0 \pm$ 4.2) $\times 10^{-13}$ day $^{-1}$. Hence, no signal could be detected associated with Saturn's $\dot{J}_{2}$, within a $1 \sigma$ uncertainty of $4.4 \times 10^{-13}$ day $^{-1}$.

Testing the influence of the inner moons' chaotic motion We tested the influence of chaos affecting the inner moons' system on our results by developing five different sets of ephemerides. The first three sets were obtained after fitting our prior model of the inner moons ${ }^{4}$ to Cassini data, and then extrapolating their orbit over more than a century to cover the full time span considered here. The first set introduced a constant step size in the integration, the second one added extra-precision in the compiling script and the last one introduced a variable step size. The fourth set of ephemerides considered extra precision with variable step size and added $H u b$ ble Space Telescope ${ }^{32}$ data in the fit. The last set was obtained similarly but adding Voyager data. While the five sets provide similar ephemeride quality during the Cassini era, they start diverging significantly before 2000. Still we found that none of the five ephemerides tested changed our results, as the differences were significantly below the error bar of the measurements. This was somewhat expected since the influence of inner moons is rather small, even on Mimas. Hence, their influence falls way below the accuracy of the measurements (Cassini data excluded). Still, it was found that their global influence was important to fit properly the 
Mimas-Tethys long term libration with Cassini data. Moreover, a proper fit of Methone requires consideration of the influence of Pandora on Mimas' orbit ${ }^{33}$.

Measuring da/dt rates and their uncertainty Since our N-body code introduces Saturn's tidal potential using the $k_{2} / Q$ parametrization, it is not systematically straightforward to get the associated variation on semi-major axis. In particular, while Eq. 1 can be used for moons outside of resonance like Rhea and Titan (Hyperion's mass is negligible enough to be disregarded), it cannot be used for Mimas, Enceladus, Tethys and Dione.

To determine the secular semi-major axis variations of these four moons, we subtracted their motion reproduced by our best solution with a similar simulation differing only by the neglect of tidal dissipation inside Saturn and Enceladus. The orbital difference between both solutions shows up as a quadratic expansion on orbital longitude, as expected by tidal theory. Then one can easily convert such quadratic longitudinal expansion into a secular rate of semimajor expansion axis using Kepler's law. The uncertainties on such variations can be obtained in the same way, this time modifying our best solution by adding/subtracting the 3- $\sigma$ uncertainties on Saturn's $k_{2} / Q$ values. Using this method we estimated the $d a / d t$ rates to be (in $\mathrm{cm} /$ year) $1.58 \pm 1.61,2.08 \pm 1.10,2.36 \pm 1.23,2.91 \pm 1.57,9.01 \pm 2.17,25.3 \pm 20.9$ for Mimas, Enceladus, Tethys, Dione, Rhea and Titan, respectively. The corresponding inverse migration timescales $t_{\text {tide }}^{-1}$ are listed in Supplementary Table 1.

\section{Data Availability}

The data that support the plots within this paper and other findings of this study are available from the corresponding author upon reasonable request. 


\section{Code availability}

All Astrometric data derived from ISS-images can be reproduced using our CAVIAR software available under Creative Commons Attribution-NonCommercial-ShareAlike 4.0 International License here: www.imcce.fr/recherche/equipes/pegase/caviar

The MONTE space navigation code was obtained through a license agreement between NASA and the Italian Space Agency; the terms do not permit redistribution. MONTE licenses may be requested at https://montepy.jpl.nasa.gov/.

The availability of NOE software is limited due to NASA restrictions.

\section{Extra References}

31. Bar-Sever, Y. E. et al. Atmospheric media calibration for the deep space network. Proceedings of the IEEE95, 21802192 (2007).

32. French, R. G. et al. Astrometry of Saturns Satellites from the Hubble Space Telescope WFPC2. The Publ. of the Astron. Soc. of the Pac. 118, 246259 (2006).

33. Jacobson, R. A. The Small Saturnian Satellites Chaos and Conundrum. InAAS/Division of Dynamical Astronomy Meeting \#45, AAS/Division of Dynamical Astronomy Meeting, 304.05 (2014).

34. Lainey, V. Quantification of tidal parameters from solar system data. Celest. Mech. and Dyn. Ast. 126, 145156 (2016). 
Figure 1 Saturnian tidal quality factor. Effective tidal quality factor $Q$ of Saturn for the tidal bulge raised by each of its moons measured in this work. Purple points are measurements from astrometric solutions, with $3 \sigma$ error bars, which extend to encompass results from each of the considered energy dissipation rates in Enceladus. Titan's red point is measured using Cassini radio tracking data. Blue shaded regions are the predicted tidal quality factors from a resonance locking model with a Saturn evolution time of $t_{p}=6$ Gyr. The horizontal dashed line is the minimum value of $Q$ that allows for coeval formation of Mimas and Saturn, assuming $Q$ is constant ${ }^{21}$. Darker background shading corresponds to a more dissipative interior of Saturn (smaller $Q$ ).

Figure 2 Tidal migration timescales. Outward migration timescales for each of Saturn's moons. Purple points are measurements from astrometric solutions as described in Methods, with $3 \sigma$ error bars, while Titan's red point is measured using Cassini radio tracking data. Blue points show the same resonance locking model as Figure , where the predicted migration time scale from resonance locking is $t_{\text {tide }} \approx 9$ Gyr for each moon, regardless of mean-motion resonances. The tidal migration timescale with a constant $Q$ (blue dashed line) corresponds to $Q=1.8 \times 10^{4}$ as in Figure. Darker background shading corresponds to faster migration (shorter migration timescale). The migration timescale of each moon is within a factor of $\approx 2$ of $10 \mathrm{Gyr}$.

Figure 3 Moon orbital evolution. A possible evolutionary history of the orbital distance of Saturn's moons as a function of time, for both a resonance locking model with inertial waves (solid colored lines) and a constant $Q=5000$ model (black dashed lines). The resonance locking models are shaded by the effective tidal quality factor, $Q_{\mathrm{ef}}$, at a given moment in time. 


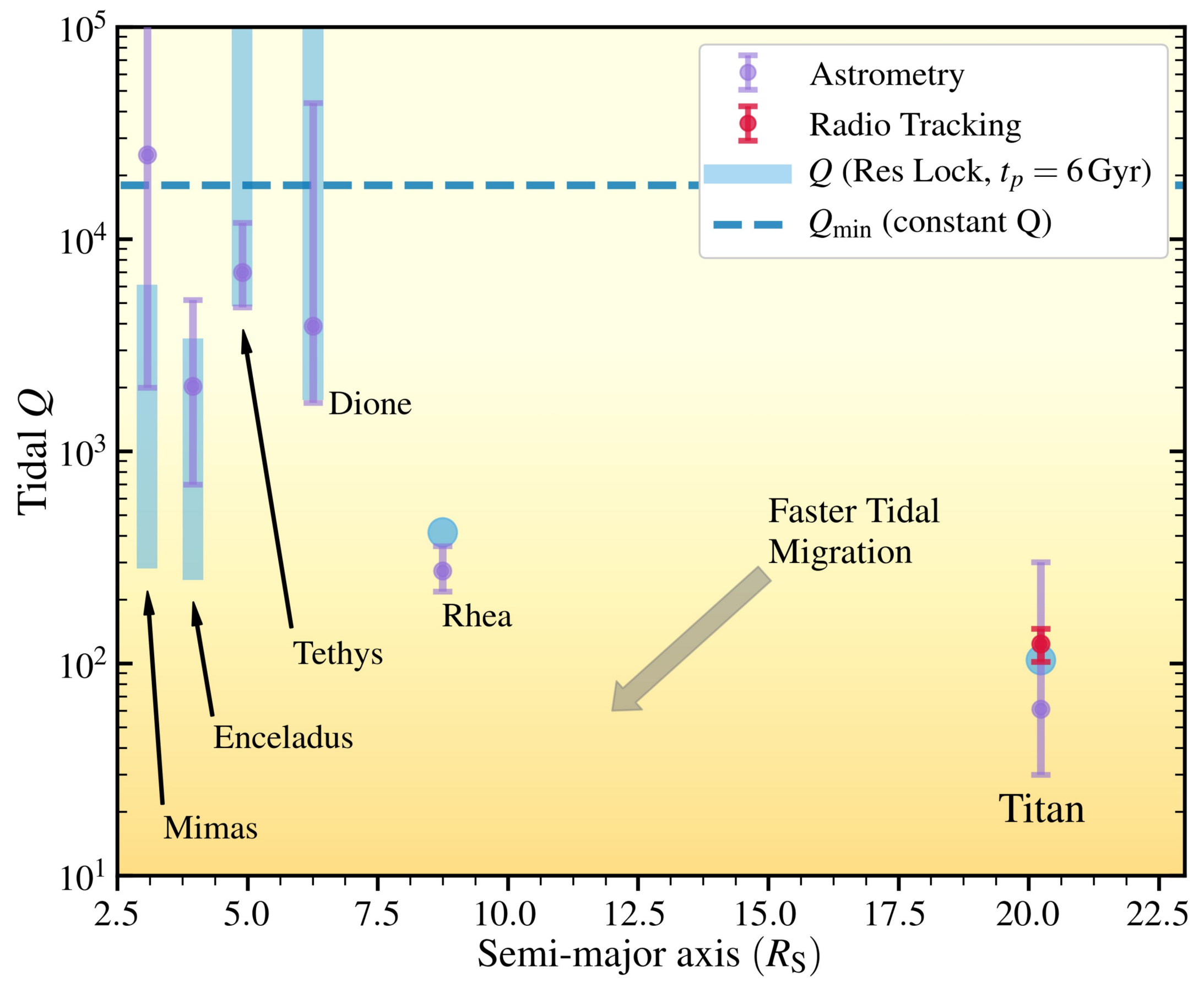




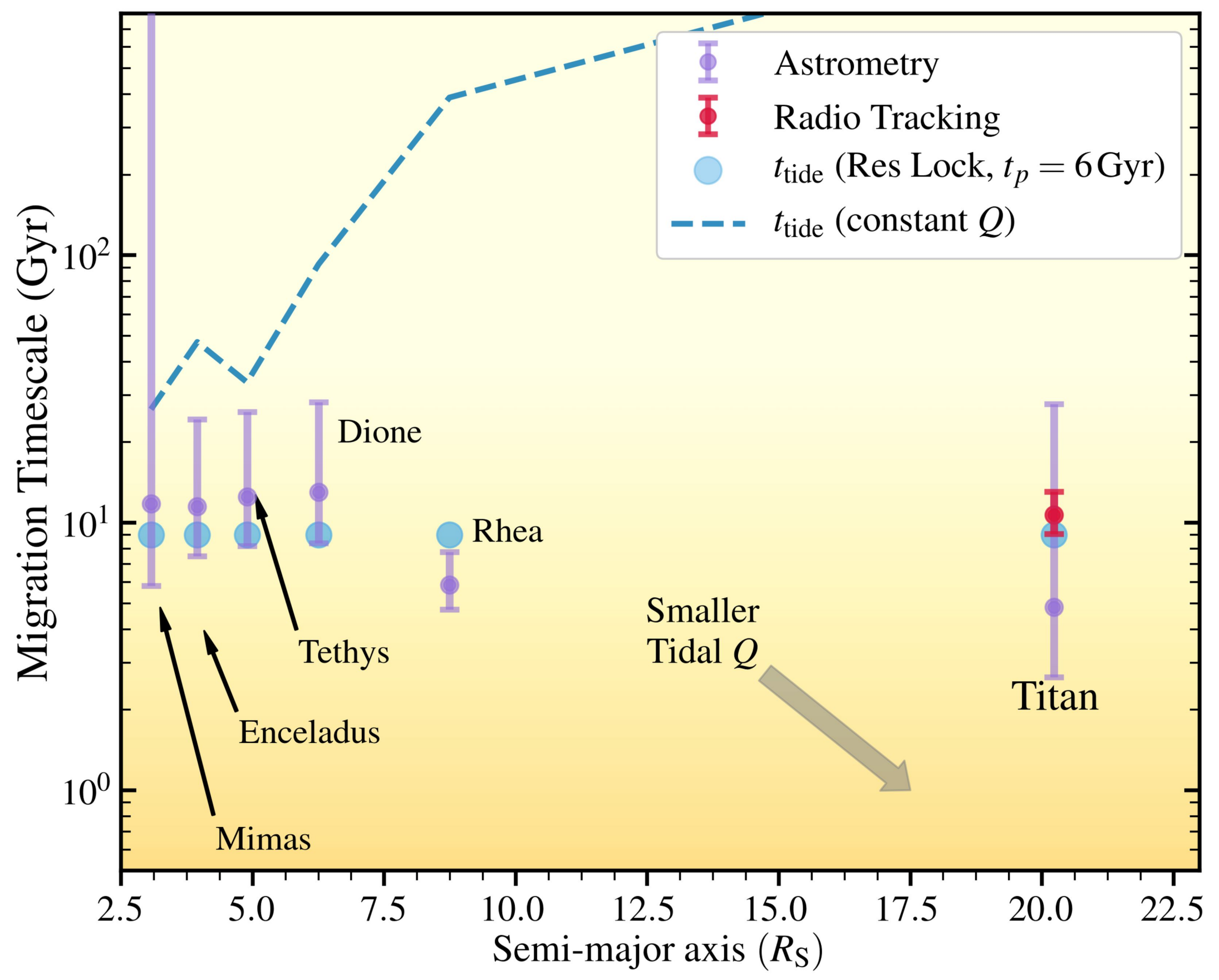

